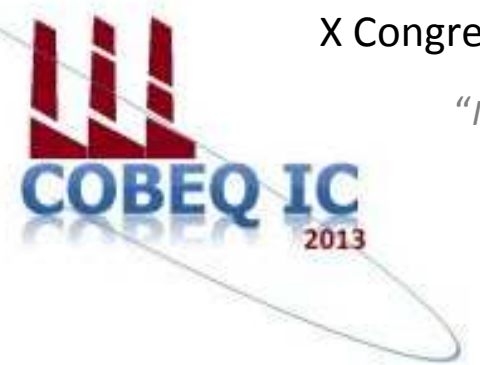

"Influência da pesquisa em Engenharia Química no desenvolvimento tecnológico e industrial brasileiro"

Universidade Federal Rural do Rio de Janeiro Universidade Severino Sombra Vassouras - RJ-Brasil

\title{
UTILIZAÇÃO DE VINHAÇA PARA A PRODUÇÃO DE POLI(3-HIDROXIBUTIRATO) POR Cupriavidus necator
}

\author{
MARTINHAGO $^{1}$ F. M.; STEFFEN ${ }^{1}$, W. F.; SANTOS ${ }^{1}$, E. C.; ZANFONATO ${ }^{2}$, K.; QUINES $^{2}$, \\ L. K. M.; SCHMIDT ${ }^{2}$, M.; GAI ${ }^{3}$, C. S.; SCHMIDELL ${ }^{4}$, W.; ARAGÃO ${ }^{4}$, G. M. F. \\ ${ }^{1}$ Aluno - EQA/CTC/UFSC ${ }^{2}$ Doutorando - EQA/CTC/UFSC \\ ${ }^{3}$ Pós-Doutorando - EQA/CTC/UFSC ${ }^{4}$ Professor - EQA/CTC/UFSC \\ Departamento de Engenharia Química e Engenharia de Alimentos - Centro Tecnológico - \\ Universidade Federal de Santa Catarina, \\ Endereço - UFSC, Bairro Trindade, Florianópolis, CEP 88040-900, SC \\ e-mail: glaucia@enq.ufsc.br
}

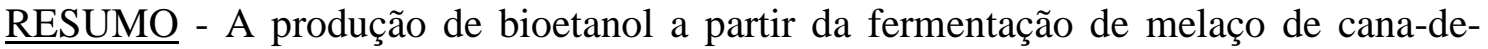
açúcar gera vinhaça como resíduo da destilação, na proporção de 4 a 101 para cada litro de produto. Geralmente usada em fertirrigação, a vinhaça causa impactos negativos no meio ambiente, contaminando solos e águas subterrâneas. Para minimizar este impacto, a vinhaça pode ser utilizada na geração de produtos com valor agregado. O Poli(3hidroxibutirato) ( $\mathrm{P}(3 \mathrm{HB})$ ) é um biopolímero, biodegradável e biocompatível, acumulado intracelularmente como reserva energética, por diversos micro-organismos (e.g. Cupriavidus necator), mas o seu elevado custo de produção o torna economicamente não competitivo. Neste contexto, a utilização da vinhaça como substrato para a produção biotecnológica de $\mathrm{P}(3 \mathrm{HB})$ é uma alternativa possível de ser explorada. Este trabalho relata a capacidade de crescimento da bactéria $C$. necator DSM 545, e produção de $\mathrm{P}(3 \mathrm{HB})$ a partir de vinhaça proveniente da destilação do mosto de cana-deaçúcar, em incubador rotativo. Os ensaios preliminares mostraram a capacidade de crescimento do micro-organismo em vinhaça não diluída sem efeito inibitório no crescimento do mesmo. Testes com a adição de minerais essenciais, fonte de nitrogênio e glicose (durante o crescimento da bactéria) à vinhaça, demonstraram a capacidade de produção de $\mathrm{P}(3 \mathrm{HB})$.
\end{abstract}

Palavras-chave: biopolímero, cana-de-açúcar, resíduo agroindustrial.

\section{INTRODUÇÃO}

Responsável por mais da metade do açúcar comercializado no mundo, o Brasil é o maior produtor de açúcar e etanol e conquista, cada vez mais, o mercado externo com o uso do biocombustível como alternativa energética (Ministério da Agricultura, Brasil, 2013). Dados publicados para a safra prevista em
2013/2014 mostram que a área plantada de cana-de-açúcar será, aproximadamente, igual a 8,8 milhões de hectares e serão produzidas 40,97 milhões de toneladas de açúcar e 27,17 bilhões de litros de etanol (CONAB, 2013).

A vinhaça, resíduo da destilação do álcool etílico produzido por via fermentativa, é também conhecida como vinhoto, restilo, caldo ou garapão (Bittencourt et al, 1978). 
A vinhaça é principalmente utilizada em fertirrigação, porém há controvérsias quanto à aplicação da vinhaça no solo, devido à possível contaminação da água subterrânea quando a mesma é aplicada em excesso (Laime et al., 2011).

A valorização da vinhaça, um resíduo rico em ácidos orgânicos, glicerol e sais minerais (Parnaudeau et al, 2008), através da sua utilização como substrato para a produção biotecnológica de Poli-hidroxialcanoatos (PHAs) é uma alternativa interessante.

Dentre os PHAs, o Poli(3hidroxibutirato) $(\mathrm{P}(3 \mathrm{HB}))$ é o biopolímero mais estudado em função de suas características similares às do polipropileno (PP), polímero de origem fóssil. Estes biopolímeros são acumulados como material de reserva de carbono e energia por vários micro-organismos, geralmente sob condições de limitação de nutrientes essenciais como nitrogênio, fósforo, enxofre ou oxigênio, e na presença de excesso de fonte de carbono (Lee et al., 1999; Khanna e Srivastava, 2005).

Muitos são os micro-organismos produtores de $\mathrm{P}(3 \mathrm{HB})$. Entre eles, a bactéria Cupriavidus necator é uma das que apresenta melhores condições à produção industrial. Esta bactéria destaca-se pela capacidade de acumular mais de $80 \%$ de sua massa seca em polímero, com alta massa molar e utilizando diferentes tipos de substratos como glicose, frutose, ácidos orgânicos dentre outros (Ramsay, 1994).

Este trabalho teve como objetivo demonstrar a capacidade de produção de $\mathrm{P}(3 \mathrm{HB})$, um biopolímero biodegradável, a partir de vinhaça, a partir de C. necator.

\section{MATERIAL E MÉTODOS}

\section{Micro-organismo e Meios de Cultivo}

O micro-organismo utilizado neste trabalho foi a bactéria Cupriavidus necator DSM 545.

Os experimentos preliminares, com o objetivo de verificar se o micro-organismo estudado teria capacidade de crescer em meio mineral (Aragão et al., 1996) com vinhaça e vinhaça adicionada de glicose $\left(20 \mathrm{~g} . \mathrm{l}^{-1}\right)$ foram realizados a partir de uma pré-cultura em caldo nutriente $(\mathrm{CN})$, que contém peptona de carne $\left(5,0\right.$ g..$\left.^{-1}\right)$ e extrato de carne $\left(3,0\right.$ g. $\left.1^{-1}\right)$. As concentrações de vinhaça durante o experimento variaram de 0,$5 ; 5 ; 10$ e $100 \%$ $(\mathrm{v} / \mathrm{v})$.

O cultivo para estudar a produção de $\mathrm{P}(3 \mathrm{HB})$ foi realizado a partir de duas préculturas, uma em $\mathrm{CN}$ e outra em meio mineral (sem adição de glicose). A adição de glicose $\left(20\right.$ g. $\left.1^{-1}\right)$ ao meio mineral foi realizada após $6 \mathrm{~h}$.

A vinhaça utilizada em todos os ensaios foi esterilizada por filtração em membrana com poro de $0,22 \mu \mathrm{m}$.

\section{Condições de Cultivo}

As pré-culturas em $\mathrm{CN}$ foram realizadas em frascos erlenmeyer aletados de $500 \mathrm{ml}$ contendo $150 \mathrm{ml}$ de meio, e permaneceram a $35^{\circ} \mathrm{C} / 150 \mathrm{rpm}$ em incubador rotativo, por $18 \mathrm{~h}$. A fração de inóculo utilizada em todos os experimentos foi de $10 \%$ (v/v).

Ensaios preliminares: Os testes foram realizados em frascos erlenmeyer aletados de $250 \mathrm{ml}$ contendo $100 \mathrm{ml}$ de meio mineral, a $35^{\circ} \mathrm{C}$ e $150 \mathrm{rpm}$ por $24 \mathrm{~h}$, em incubador rotativo.

Cultivo: O cultivo foi conduzido em frasco erlenmeyer aletado de $1000 \mathrm{ml}$ com $200 \mathrm{ml}$ de meio, a $35^{\circ} \mathrm{C} / 150 \mathrm{rpm}$ por $26 \mathrm{~h}$, em incubador rotativo, sendo que, este partiu de uma segunda pré-cultura, também em meio mineral, que permaneceu sob as mesmas condições por $6 \mathrm{~h}$.

\section{Técnicas Analíticas}

Amostragem: Amostras de $2 \mathrm{ml}$ foram coletadas a cada $2 \mathrm{~h}$ e acondicionadas em microtubos de plástico e centrifugadas (10956 $\mathrm{xg} / 3 \mathrm{~min}$ ). Os sobrenadantes e os precipitados foram congelados separadamente para posteriores análises.

Determinação da concentração da biomassa: $\mathrm{O}$ acompanhamento do crescimento da biomassa foi realizado por espectrofotometria $A_{600 \mathrm{~nm}}$ e por meio de análise gravimétrica, através da filtração de $5 \mathrm{ml}$ de meio cultivado em membrana de celulose com $0,22 \mu \mathrm{m}$ de poro, em seguida as membranas, previamente taradas, foram secas e pesadas.

Determinação da concentração de glicose e glicerol: As amostras foram 
analisadas por cromatografia líquida de alta pressão (HPLC) em um equipamento Jasco (LC-2000Plus Series). Os picos analisados foram detectados com um detector de índice de refração (RI-2031 - Jasco).

Determinação demanda química de oxigênio (DQO): Foi realizada segundo o procedimento do Standart Methods for the Examination of Water and Wastewater (APHA, AWWA, WEF, 1995).

Determinação da concentração de $\underline{\mathrm{P}(3 \mathrm{HB})}$ : As células contendo $\mathrm{P}(3 \mathrm{HB})$ foram analisadas por HPLC, segundo Karr et al. (1983) com modificações. Esta análise foi realizada em equipamento Jasco (LC-2000Plus Series). A concentração foi detectada a $\mathrm{A}_{210 \mathrm{~nm}}$ (Jasco UV-2077).

\section{Tratamento dos Dados}

Considerando-se que o acúmulo de $\mathrm{P}(3 \mathrm{HB})$ é intracelular, é importante a definição de: $\mathrm{P}(3 \mathrm{HB})$;

$\mathrm{Xt}($ biomassa total $)=$ biomassa contendo

$\mathrm{Xr}($ biomassa residual $)=\mathrm{Xt}-\mathrm{P}(3 \mathrm{HB})$.

Velocidade específica de crescimento da biomassa: A partir dos perfis de concentração celular, foi possível determinar as velocidades instantâneas de crescimento microbiano (dXr/dt). Dividindo-se estas velocidades pela concentração celular residual no instanet $t$, a velocidade específica de crescimento celular $\left(\mu_{\mathrm{Xr}}\right)$ foi obtida e está representada pela Equação 1.

$\mu_{\mathrm{X}_{\mathrm{r}}}=\frac{1}{\mathrm{Xr}} \frac{\mathrm{dXr}}{\mathrm{dt}}$

Os ajustes aos dados experimentais foram realizados com o auxílio do software Microsoft Office Excel 2007.

\section{RESULTADOS E DISCUSSÃO}

Os ensaios preliminares, realizados com o objetivo de verificar o possível efeito inibitório da vinhaça no crescimento de C. necator, demonstraram que, sob as condições estudadas, houve crescimento da biomassa em todas as concentrações testadas $(0,50$ a $100 \%)(\mathrm{v} / \mathrm{v})$. Conforme Bekatorou et al. (2006) uma das vantagens da utilização da vinhaça em processos biotecnológicos, é a mesma ser livre de toxinas e inibidores de fermentação. Por outro lado, Santos et al. (2003) afirmaram que a presença de compostos, como os compostos fenólicos, podem ser tóxicos e/ou inibir microorganismos.

Estudos reportados da literatura que avaliaram variações da concentração de vinhaça no crescimento de micro-organismos, normalmente leveduras, como Candida lipolytica, Rhodotorula mucilaginosa, Saccharomyces cerevisiae, Candida parapsilosis, utilizaram concentrações de vinhaça de até $75 \%$ (v/v) e com adição de outros nutrientes como peptona, extrato de levedura, melaço e também glicose (Silva et al., 2011; Cazetta e Celligoi, 2006).

Com a comprovação do crescimento de C. necator em vinhaça pura $(100 \%-\mathrm{v} / \mathrm{v})$ apenas com adição de sais minerais, ensaios com diferentes estratégias de cultivo (dados não apresentados) foram realizados com $\mathrm{o}$ objetivo de se estabelecerem as condições experimentais. A partir das respostas obtidas nesses testes, foi possível concluir que, o principal inconveniente da utilização de vinhaça para a produção de $\mathrm{P}(3 \mathrm{HB})$, é sua baixa concentração da fonte de carbono, apesar dos índices de DQO de 30 a 100 g..$^{-1}$. (Pant e Adholeya, 2007; Jimenez et al., 2006; Beltran et al., 2005).

Como este trabalho se propôs a analisar também a produção de $\mathrm{P}(3 \mathrm{HB})$ em frascos agitados, uma adição complementar de vinhaça ao longo do cultivo, com a finalidade de aumentar a fonte de carbono disponível, não seria conveniente, visto a grande variação de volume que seria acarretada. Desta forma, para permitir o crescimento de $C$. necator em vinhaça pura (com adição de sais) e o acúmulo de biopolímero, adotou-se a estratégia de adição de uma solução concentrada de glicose, para elevar esta concentração a aproximadamente $20 \mathrm{~g} . \mathrm{l}^{-1}$, ao final da fase exponencial de crescimento.

A Figura 1 apresenta os dados de concentração total de células (Xt), de concentração residual $(\mathrm{Xr})$ e de concentração de biopolímero (P3HB)) ao longo do cultivo. 


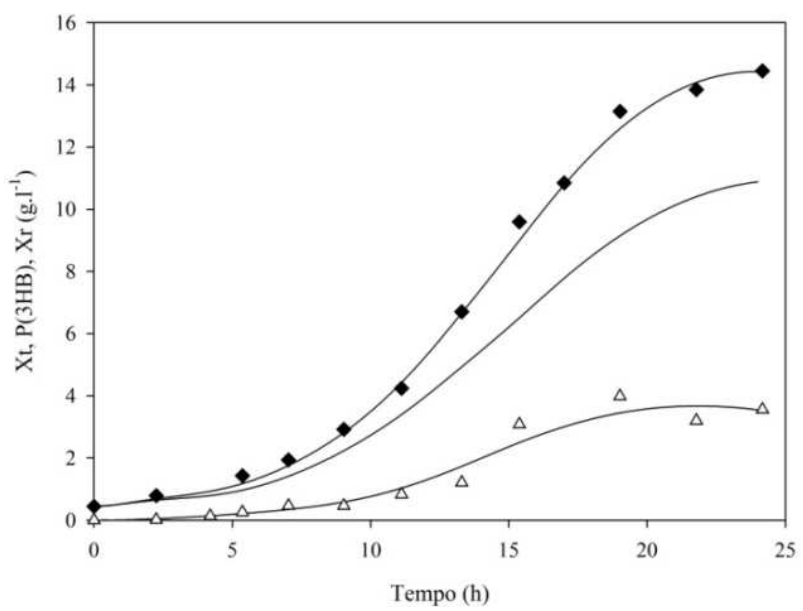

Figura 1 - Evolução da biomassa total ( $\diamond)$ biomassa residual (-) e da produção de $\mathbf{P}(3 \mathrm{HB})(\mathbf{\Delta})$, ao longo do cultivo. A linha contínua representa os ajustes feitos pelo software Microsoft Office Excel (2007).

A porcentagem final de biopolímero acumulado foi de $30 \%(\mathrm{p} / \mathrm{v})$ e a velocidade específica máxima de crescimento celular $\left(\mu_{\mathrm{Xr}}\right)$ foi de $0,20 \mathrm{~h}^{-1}$. O acúmulo de $\mathrm{P}(3 \mathrm{HB})$ foi relativamente baixo quando comparado a outros estudos da literatura, com valores próximos a $80 \%$ de $\mathrm{P}(3 \mathrm{HB})$, dependendo das condições experimentais (Reinecke e Steinbüchel, 2009). Porém, o valor de $\mu_{\mathrm{Xr}}$ encontrado está de acordo com a literatura consultada para este micro-organismo (Khan et al., 2013; Tavares et al., 2004).

Pramanik et al. (2012) utilizaram vinhaça para a produção de $\mathrm{P}(3 \mathrm{HB})$ a partir de Haloarcula marismortui, um micro-organismo halofílico (capaz de crescer em elevadas concentrações de sais) e o acúmulo final de $\mathrm{P}(3 \mathrm{HB})$ em meio com $10 \%$ de vinhaça foi de $30 \%$ com $\mu_{\mathrm{X}}$ de $0,086 \mathrm{~h}^{-1}$. Myshkina et al. (2008) com o objetivo de produzir $\mathrm{P}(3 \mathrm{HB})$ a partir de vinhaça $(25 \%$ - v/v) e outros resíduos, obtiveram acúmulo de $73 \%$ utilizando Azotobacter charoococcum. Sendo os trabalhos citados, uns dos poucos encontrados contendo relatos de produção de $\mathrm{P}(3 \mathrm{HB})$ a partir de vinhaça, independente do micro-organismo utilizado. Cabe ressaltar que neste estudo a vinhaça não foi diluída, o que torna o acúmulo encontrado de biopolímero bastante satisfatório.

$\mathrm{Na}$ Figura 2 são apresentados os dados de concentração residual de glicose, glicerol, DQO e nitrogênio, todos em g. $1^{-1}$.

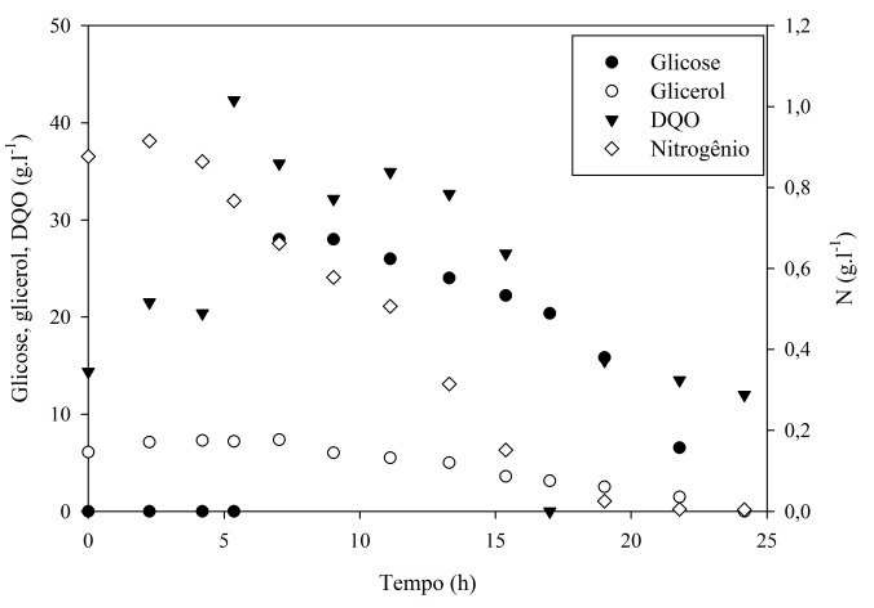

Figura 2 - Dados de concentração residual de glicose $(\bullet)$, glicerol $(\circ)$, DQO $(\nabla)$ e nitrogênio $(\diamond)$, ao longo do cultivo.

É possível observar na Figura 2 que antes da adição de glicose (aproximadamente 6 h) esta não se encontrava disponível no meio, como substrato acredita-se que o microorganismo tenha assimilado outras fontes de carbono (não quantificadas neste estudo) presentes na vinhaça, como por exemplo, os ácidos orgânicos (Robles-González et al., 2012; Elia Neto e Nakahondo, 1995), comprovada através dos níveis de DQO que se encontravam em torno de 20 g. $1^{-1}$.

Informações disponíveis na literatura sugerem que um dos maiores componentes orgânicos da vinhaça da cana-de-açúcar é o glicerol (Parnaudeau et al., 2008), porém foi observado que o glicerol proveniente da vinhaça, parece ter sido consumido somente após a adição da glicose.

O nutriente utilizado como limitante neste estudo, para o processo de síntese de $\mathrm{P}(3 \mathrm{HB})$, foi o nitrogênio e sua limitação pode ser observada (Figura 2) em torno de $12 \mathrm{~h}$ de cultivo e sua exaustão em aproximadamente $18 \mathrm{~h}$, permitindo assim o acúmulo de $\mathrm{P}(3 \mathrm{HB})$, que como mostrado na Figura 1, começou a aumentar neste instante de tempo.

Ao final do cultivo, a DQO se encontrava em aproximadamente $15 \mathrm{~g} . \mathrm{l}^{-1}$, e a biomassa total estava entrando em fase de decaimento, sugerindo que, da DQO disponível na vinhaça, determinados compostos não são capazes de serem consumidos pelo micro-organismo estudado.

Frente ao exposto, este estudo demonstrou a capacidade de produção de 
$\mathrm{P}(3 \mathrm{HB})$ a partir de vinhaça, sem utilização de pré-tratamento, apenas esterilização.

A produção deste biopolímero a partir de vinhaça pode ser promissora tanto do ponto de vista ambiental, como econômico, visto os elevados custos de produção de PHAs.

\section{CONCLUSÃO}

C. necator foi capaz de produzir $\mathrm{P}(3 \mathrm{HB})$ a partir de um resíduo abundante e poluente. Este é o primeiro relato de produção de biopolímero por este micro-organismo utilizando vinhaça pura.

\section{REFERÊNCIAS}

APHA, AWWA, WEF. (1995), Standard methods for the examination of water and wastewater $19^{\text {th }}$. American public health association. Washington, DC.

ARAGÃO, G. M. F., LINDLEY, N. D., URIBELARREA, J. L., PAREILLEUX, A. (1996), "Maintaining a controlled residual growth capacity increases the production of polyhydroxyalkanoate copolymers by Alcaligenes eutrophus". Biotechnology Letters, 18, 937-942.

BEKATOROU, A.; PSARIANOS, C.; KOUTINAS, A. (2006), "Production of food grade yeasts". Food Technology and Biotechnology, 44, 407-415.

BELTAN, J.; DOMINGUEZ, J.; PARTIDO, E. (2005), "Phisico-chemical treatment for the depuration of wine distillery wastewaters (vinasses)". Water Science and Technology, 51, 159-166.

BITTENCOURT, V. C., CASTRO, L. J. B., FIGUEIREDO, A. A. M., PAIXÃO, A. C. S., POLLI, D. M. (1978), "Composição da Vinhaça”. Brasil Açucareiro, 92, 25-35.

CAZETTA, M. L.; CElligoI, M. A. P. C. (2006), "Study of molasses/vinasse waste ratio for single cell protein and total lipids production by microorganisms". Semina: Ciências Exatas e Tecnológicas, Londrina, 27, 03-10.
CONAB - COMPANHIA NACIONAL DE ABASTECIMENTO. Acompanhamento de safra brasileira: cana-de-açúcar estimativa safra 2013/2014.

ELIA NETO, A.; NAKAHONDO, T. (1995), Caracterização físico-química da vinhaça projeto $\mathrm{N}^{\circ}$ 9500278. Relatório técnico da seção de tecnologia de tratamento de águas do centro de tecnologia coopersucar, Piracicaba, $26 \mathrm{p}$.

JIMÉNEZ, A. M.; BORJA, R.; MARTÍN, A.; RAPOSOB, F. (2006), "Kinectic analysis of the anaerobic digestion of untreated vinasses and vinasses previously treated with Penicillium decumbengs". Journal of Environmental Management, 80, 303-310. KARR, D. B.; WATERS, J. K. A; EMERICH, D. W. (1983), "Analysis of Poly- $\beta$ Hydroxybutyrate in Rhizobium japonicum Bacteroids by Ion-Exclusion HighPressure Liquid Chromatography and UV detection". Applied and Environmental Microbiology, 46, 1339-1344.

KHAN, M. R.; PRASAD, E. N. R.; ABDULLAH, H; BATCHA, A. F. M. (2013), "Kinetic analysis on cell growth and biosynthesis of poly(3hydroxibutyrate) (PHB) in Cupriavidus necator H 16". Journal of Bioscience, Biochemistry and Bioinformatics, 3, 516519.

KHANNA, S.; SRIVASTAVA, A. K. (2005), "Recent advances in microbial polyhydroxyalkanoates". Process Biochemistry, 40, 609-619.

LAIME, E. M. O., FERNANDES, D. C. S., FREIRE, E. A. (2011), "Possibilidades tecnológicas para a destinação da vinhaça: uma revisão". Revista Trópica - Ciências Agrárias e Biológicas, 5, 16.

LEE, S. Y., CHOI, J., WONG, H. H. (1999), "Recent advances in polyhydroxyalkanoate production by bacterial fermentation: mini-review". International Journal of Biological Macromolecules, 25, 31-36. 
MINISTÉRIO DA AGRICULTURA (2013), http://www.agricultura.gov.br/vegetal/cult uras/cana-de-acucar, acessado em 28 de agosto de 2013.

MYSHKINA, V. L.; NIKOLAEVA, D. A.; MAKHINA, T. K.; BONARTSEV, A. P.; BONARTSEVA, G. A. (2008), "Effect of growth conditions on the molecular weight of Poly-3-hydroxybutyrate produced by Azotobacter chroococcum 7B" Applied Biochemistry and Microbiology, 44, 482-486.

PANT, D.; ADHOLEYA, A. (2007), "Biological approaches for treatment of distillery wastewater: a review". Bioresource Technology, 98, 2321-2334.

PARNAUDEAU, V.; CONDOM, N.; OLIVER, R.; CAZEVIEILLE, P.; RECOUS, S. (2008), "Vinasse organic matter quality and mineralization potential, as influenced by raw material, fermentation and concentration process". Bioresource Technology, 99, 1553-1562.

PRAMANIK, A.; MITRA, A.; ARUMUGAM, M.; BHATTACHARRYYA, A.; SADHUKHAN, S.; RAY, A.; HALDAR, S.; MUKHOPADHYAY, U. K.; MUKHERJEE, J. (2012), "Utilization of vinasse for the production of polyhydroxybutyrate by Haloarcula marismortui". Folia Microbiology, 57, 71-79.

RAMSAY, B.A. (1994), "Physiological factor affecting PHA production. Physiology, kinetics, production and use of biopolymers". Proceedings, 9-7, Austria.

REINECKE, F., STEINBÜCHEL, A. (2009). "Ralstonia eutropha strain H16 as model organismfor PHA metabolism and for biotechnological production of technicallyinteresting biopolymers". Journal of Molecular Microbiology and Biotechnology, 16, 91-108.

ROBLES-GONZÁLEZ， V.; GALÍNDEZMAYER, J.; RINDERKNECHT-SEIJAS, N.; POGGI-VARALDO, H. M. (2012),
"Treatment of mezcal vinasses: A review". Journal of Biotechnology, 157, 524-546.

SANTOS, M. A. M., BOCANEGRA, J. L. F., MARTÍN, A. M., GARCÍA, I. G. (2003) "Ozonation of vinasse in acid and alkaline media". Journal of Chemical Technology and Biotechnology, 78, 1121-1127.

SILVA, C. F.; ARCURI, S. L.; CAMPOS, C. R.; VILELA, D. M.; ALVES, J. G. L. F.; SCHWAN, R. F. (2011), "Using the residue of spirit production and bioethanol for protein production by yeasts". Waste Manegement, 31, 108-114.

TAVARES, L. Z., SILVA, L. S., PRADELLA, J. G. C. (2004), "Production of poly(3-hydroxybutyrate) in an airlift bioreactor by Ralstonia eutropha". Biochemical Engineering Journal, 18, 2131. 